\title{
CFTR is required for the migration of primordial germ cells during zebrafish early embryogenesis
}

\author{
Huijuan Liao,*, Yan Chen,*, Yulong Li¹,2, Shaolong Xue ${ }^{1,3}$, Mingfeng Liu, ${ }^{1,4}$ Ziyuan Lin, \\ Yanyan Liu ${ }^{5}$, Hsiao Chang Chan ${ }^{1,3}$, Xiaohu Zhang ${ }^{1}$ and Huaqin Sun ${ }^{1}$ \\ ${ }^{1}$ SCU-CUHK Joint Laboratory for Reproductive Medicine, Key Laboratory of Birth Defects and Related Diseases of \\ Women and Children (Sichuan University), Ministry of Education, Department of Pediatrics, West China Second \\ University Hospital, Sichuan University, Chengdu, People's Republic of China, ${ }^{2}$ The School of Life Science, \\ Shandong University, Jinan, Shandong, People's Republic of China, ${ }^{3} E$ pithelial Cell Biology Research Center, School \\ of Biomedical Sciences, Faculty of Medicine, The Chinese University of Hong Kong, Hong Kong SAR, People's \\ Republic of China, ${ }^{4}$ Key Laboratory of Bio-Resource and Eco-Environment of Ministry of Education, College of Life \\ Science, Sichuan University, Chengdu, Sichuan, People's Republic of China and ${ }^{5}$ Prenatal Diagnosis Center, \\ Department of Obstetrics \& Gynecologic, Key Laboratory of Birth Defects and Related Diseases of Women and \\ Children (Sichuan University), Ministry of Education, West China Second University Hospital, Sichuan University, \\ Chengdu, People's Republic of China
}

Correspondence should be addressed to H Sun or X Zhang; Email: sunhuaqin@scu.edu.cn or zhangxh_alex@163.com

$*(\mathrm{H}$ Liao and $\mathrm{Y}$ Chen contributed equally to this work)

\begin{abstract}
Mutations in the cystic fibrosis transmembrane conductance regulator (CFTR) gene affect fertility in both sexes. However, the involvement of CFTR in regulating germ cell development remains largely unknown. Here, we used zebrafish model to investigate the role of CFTR in primordial germ cells (PGCs) development. We generated a cftr frameshift mutant zebrafish line using CRISPR/Cas9 technique and investigated the migration of PGCs during early embryo development. Our results showed that loss of Cftr impairs the migration of PGCs from dome stages onward. The migration of PGCs was also perturbed by treatment of CFTRinh-172, a gatingspecific CFTR channel inhibitor. Moreover, defected PGCs migration in cftr mutant embryos can be partially rescued by injection of WT but not other channel-defective mutant cftr mRNAs. Finally, we observed the elevation of cxcr4b, cxcl12a, rgs14a and ca15b, key factors involved in zebrafish PGCs migration, in cftr-mutant zebrafish embryos. Taken together, the present study revealed an important role of CFTR acting as an ion channel in regulating PGCs migration during early embryogenesis. Defect of which may impair germ cell development through elevation of key factors involved in cell motility and response to chemotactic gradient in PGCs.

Reproduction (2018) 156 261-268
\end{abstract}

\section{Introduction}

Cystic fibrosis transmembrane conductance regulator (CFTR) is a cAMP-activated anion channel belongs to ATP-binding cassette (ABC) transporter superfamily (Gadsby et al. 2006). Mutations of CFTR cause cystic fibrosis (CF), the most common lethal congenital disease in Caucasians (Quinton 1999, Riordan 2008). The most well-characterized mutation $\Delta \mathrm{F} 508$ affects the trafficking and maturation of CFTR. Another common mutation G551D impairs CFTR channel gating and markedly reduces channel opening probability (Welsh \& Smith 1993, Hwang \& Kirk 2013). Regardless of the underlying mechanisms, the net outcome of these mutations is the diminished ion channel function of CFTR (Welsh \& Smith 1993, Hwang \& Kirk 2013).
The hallmark of CF is the defects in electrolyte and fluid transport that affect multiple organ systems with a multitude of clinical manifestations (Quinton 1999, Riordan 2008). The reproductive tract is one of the major systems being affected by CFTR mutation. Most CF men are infertile due to anatomical abnormalities of the reproductive tract. Besides, CFTR in sperm may be involved in the transport of $\mathrm{HCO}_{3}-$, which is important for sperm capacitation, and CFTR mutations with impaired CFTR function may lead to reduced sperm fertilizing capacity and male infertility (Xu et al. 2007). Women with CF have anatomically normal reproductive tracts. Nonetheless, subfertility and infertility are still observed in CF women due to other factors such as ovulation failure. CFTR regulates ovarian estrogen biosynthesis by amplifying the FSH-stimulated signal 
via the nuclear soluble adenylyl cyclase (sAC), defective CFTR-dependent regulation of estrogen production may underlie the ovarian disorders seen in CF and polycystic ovarian syndrome (PCOS) (Chen et al. 2012). Despite the importance of CFTR in the reproductive system, little is known about the role of CFTR in germ cell development.

CFTR is expressed in germ cells of various developmental stages. In the testis, CFTR localized in the cytoplasm and plasma membrane of differentiated germ cells. CFTR and sAC are involved in regulating the CAMP-CREB signaling pathway in Sertoli cells, defect of which may result in impaired spermatogenesis and azoospermia (Snouwaert et al. 1992, Xu et al. 2011). Besides the roles in differentiated germ cells, CFTR also plays an important role in embryo development as an ion channel (Lu et al. 2012, 2016). Intriguingly, apart from its ion channel function, CFTR can also serve as a protein interaction hub and modulate the differentiation of embryonic stem cells via its interaction with $\beta$-catenin (Liu et al. 2017). The demonstrated roles of CFTR in primitive stem cells and germ cells prompted us to investigate the potential involvement of CFTR in regulating the development of the most primitive type of germ cells, the primordial germ cells (PGCs).

PGCs are the primary undifferentiated stem cell type, which are formed in a distinct position from where the gonad develops at an earlier time and actively migrate to the gonadal ridge during early embryogenesis (Weidinger et al. 1999, 2002). The migration of PGCs is regulated by both attractive and repulsive guidance cues established by the somatic cells along the migration path (Paksa \& Raz 2015). The number of PGCs arrived the gonad is correlated with sex determination and the onset of oogenesis or spermatogenesis (Nikolic et al. 2016). Depletion of PGCs in zebrafish favors testis formation. However, the testis development of PGC-depleted gonads appears to be restrained and delayed, suggesting that PGCs number may directly regulate the variability and length of gonadal transformation and testicular differentiation in zebrafish (Tzung et al. 2015).

In zebrafish, the PGCs can be readily detected by marker genes and the migration process is completed within the first day of development. Hence, the zebrafish is an excellent in vivo model for investigating the migration of PGCs (Sang et al. 2008). Here, we used zebrafish model and two PGCs markers vasa and nanos 1 to investigate the function of cftr in PGCs during embryo development. Our results showed that the localization of PGCs was impaired in cftr mutant embryos, suggesting an important role of $\mathrm{cftr}$ in regulating PGCs migration during early embryo development.

\section{Materials and methods}

\section{Ethics statement}

All experiments in this study were in accordance with the 'Guide for the Care and Use of Laboratory Animals' (Eighth Edition, 2011. ILARCLS, National Research Council, Washington, D.C.) and were approved by the Animal Care and Use Committee of West China Second University Hospital, Sichuan University (Approval ID: HXDEYY20131021).

\section{Zebrafish and embryos}

Zebrafish WT embryos from AB strain were used. Embryos were obtained by natural mating and cultured in embryo medium (Westerfield 1993). Staging of the embryos was carried out according to Kimmel et al. (Kimmel et al. 1995).

\section{Cas9/gRNA-mediated cftr mutagenesis in zebrafish}

The guide RNA and cas9 plasmid pair was kindly provided by Prof. Bo Zhang (Peking University), and the mutagenesis was performed as described by Chang et al. and Liu et al. (Chang et al. 2013, Liu et al. 2014). The cftr mutant embryos were obtained by mating heterozygous cftr mutant fishes. Mutant lines were bred for three generations to minimize any off-target effects from the genome editing, and we studied the F4 generation for PGCs consequences.

\section{Reagents and constructs}

CFTRinh-172 (Catalog No. S7139) was from Selleck. At the beginning of blastula period (2.5 hpf), embryos (30 embryos in a well of six-well plate with $3 \mathrm{~mL}$ culture water) were treated with CFTRinh-172 for $2 \mathrm{~h}$ and then subject to whole-mount in situ hybridization. The following antibodies were used: antiCFTR (Abcam, ab2784) and anti- $\beta$-tubulin (Epitomics, 1879-1 and Zen Bioscience, 200608).

WT CFTR plasmid is kept in our lab, $\Delta$ F508, G551D and $1556 \mathrm{~V}$ mutant plasmids of CFTR were constructed using KOD-Plus-Mutagenesis Kit (TOYOBO). Fragments of PGCs marker genes vasa (GenBank\# NM_131057, primer 'CGCGGATCCAGATCAGAGTCCCGTTGTGTCTTGC' and 'CCGGAATTCCTCTGCCTTCTCCTCCCTCATCGTT') and nonos1 (GenBank\# NM_131878, primer 'CCGGAATTCTGGTGGACAAGAACTACTGCTCGGT' and 'CGCGGATCCTTCCTCACATTTTTCACTCCATCAC') were cloned into vector pSPT18 (Roche, DIG RNA Labeling Kit) for antisense RNA probe synthesis.

\section{RNA and microinjection}

Capped mRNAs were synthesized using mMESSAGE mMACHINE Kit (Ambion). Synthetic capped mRNAs were injected into single-cell embryos. Injection dose was an estimated amount received by a single embryo, $~ 50 \mathrm{pg}$ mRNA were injected into embryos. 


\section{Zebrafish embryo in situ hybridization}

Whole-mount in situ hybridization (WISH) was carried out as previously described by Thisse et al. (Thisse \& Thisse 2008) and Sun et al. (Sun et al. 2010). After cleavage by appropriate restriction enzymes, antisense RNAs for in situ hybridization were synthesized using DIG RNA Labeling Kit (SP6/T7) (Roche) and purified by MEGAclear (Ambion). Antisense RNAs probe template of ca15b was amplified from zebrafish genome by primer 'TCTACATCAACAACTCCAGCAA' and

'GAAATTAATACGACTCACTATAGGG AGACCCGTGACAAGTGAAAACCCACAAT'.

\section{Quantitative real-time RT-PCR (qPCR) analysis and statistics}

Total RNA of each sample was prepared with TRIzol (Invitrogen, 15596-018) from pooled 50 embryos and cDNA was synthesized from $1 \mu \mathrm{g}$ of RNA with PrimeScript RT reagent Kit (Takara, DRR037A). qPCR with three independent biological replicates and three technical replicates was performed with the SYBR Green detection method with 7500 real-time PCR system (Applied Biosystems). The primers used to detect cxcr4b were 'GCAGGCTTGAAGGAATTCGG' and 'ATTGCTGACTGAGAGGTCGC'; rgs14a were 'TCACCTGTTTGAATTTGAGGCG' and 'GAAACCGCCTGAGTCTGACA'; were 'CTGTCACAGTTGCTCCTGGAT' and 'GGCTTGGCGTTGGAAATCG'; ca15b were 'TCAGGCTCCGTTTCATGGTG' and 'TTGGAGACTGTTGAGTGCCG'; $\beta$-actin (housekeeping gene) were 'ATGAGTCTGGCCCATCCATC' and 'CCTTTGCCAGTTTCCGCATC'. All detected genes expression was relative to $\beta$-actin. Quantitative data show mean + S.D. The comparative CT (cycle threshold) method (also known as $\Delta \Delta \mathrm{CT}$ method) was used to analyze the data. Statistical significance is defined as $P<0.05(*), P<0.01\left({ }^{* *}\right), P<0.001\left({ }^{* * *}\right)$.

\section{Results}

\section{Ectopic localization of PGCs in cftr-mutant zebrafish embryos}

Similar to the expression profile in mouse embryos, cftr was ubiquitously expressed in zebrafish embryos (Sun et al. 2018). To investigate the potential involvement of Cftr in PGCs development, we inserted mutations near the start codon of Cftr using CRISPR/Cas9 system (Fig. 1). After screening Cas9/gRNA-injected zebrafish, we identified a heterozygous line that carried a 9-bp deletion (Fig. 1A), which was predicted to delete the start codon of Cftr (Fig. 1A and Supplementary Fig. 1, see section on supplementary data given at the end of this article). We obtained heterozygous male and female fishes by back-crossing the founder with WT AB strain. The homozygous cftr-mutant fishes were obtained by crossing the heterozygous lines and screening the offspring. Consistent with previous report (Navis et al. 2013), our cftr homozygous mutant embryos also demonstrated the absence of Kupffer's vesicle (KV) lumen at 8-somite stage (Fig. 1E). We also found that a large percentage of homozygous cftr-mutant larvae was lost beginning around $10 \mathrm{dpf}$ (Navis \& Bagnat 2015). Similar to our previous work (Sun et al. 2018), we used heterozygous cftr mutant line instead in this study, and the offspring of the heterozygous crosses (mutant line)

\section{A} WT ACTtTTtAgGATGCAgAGATCACCTGTGGAGgATGCCAAC cftr mutant ACTTTTTAGGAT-_-ACCTGTGGAGGATGCCAAC Destroyed start codon

B

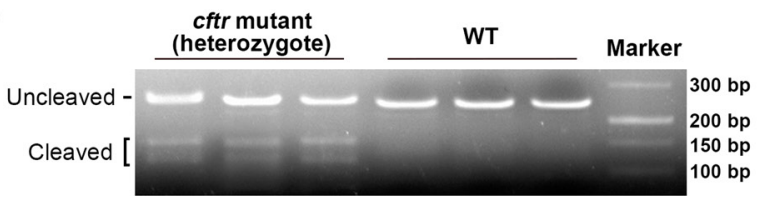

C
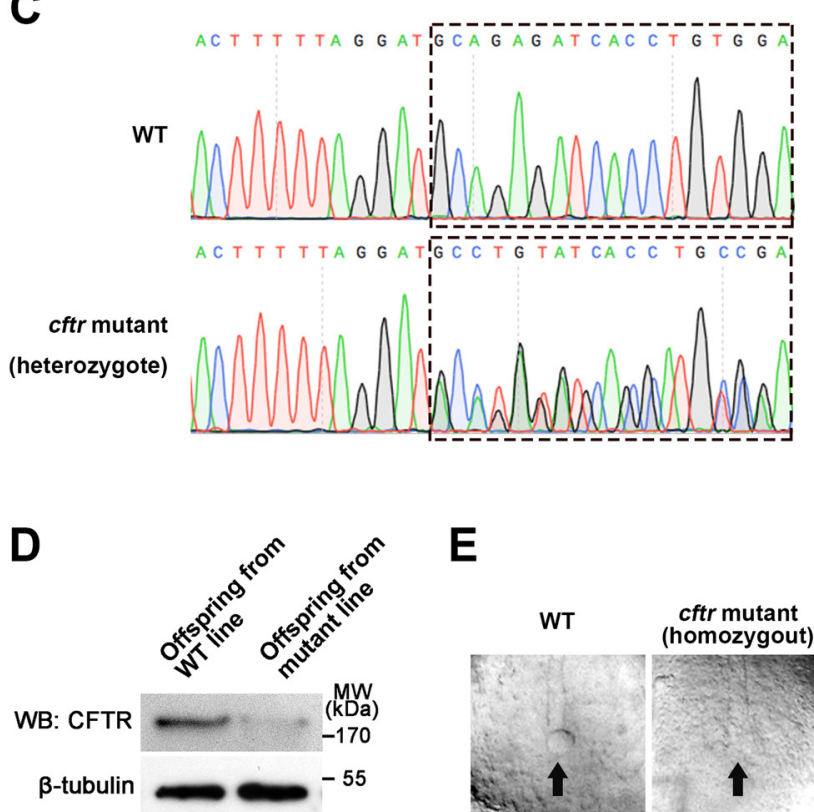

E

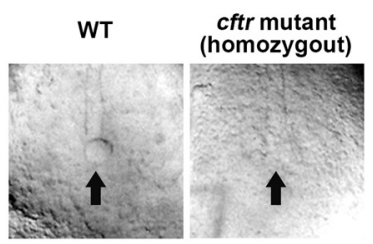

Figure 1 Targeted indel mutation induced by engineered Cas9/gRNA at the cftr gene in zebrafish. (A) The target sites highlighted by yellow and the PAM sequence marked by red underlined text. Deletions of cftr mutant are shown as dashes. Boxes show the start codon of WT and destroyed start codon of cftr mutant. (B) Gel shows T7E1 digestion of PCR products amplified from adult tail genomic DNA of F1 heterozygous generation. The uncleaved and cleaved PCR products are indicated. After digestion with T7E1, the cleaved PCR product of the adult tail represents the fragments containing the mutation. (C) Sequencing results show that F1 heterozygous generation fish carrying $\mathrm{ctt}$ mutant produces overlapping peaks marked by dashed box. (D) Western blot assay indicates the significant reduced Cftr protein level in offspring embryos from mutant line. (E) The got genotyped homozygous cftr mutant embryo also demonstrated the absent of Kupffer's vesicle (KV) lumen (pointed by arrow) at 8-somite stage. 
could be WT $(25 \%)$, heterozygous cftr+/- $(50 \%)$ and homozygous cftr-/- mutants (25\%).

Next, we compared the PGCs manifestation in offspring embryos from WT and cftr mutant line. PGCs are marked by nanos 1 and vasa at 4-cell, Dome, 50\% epiboly, 8-somite and Prim-5 stage (Raz 2003). Therefore, we examined the development of PGCs by detecting these two marker genes through WISH. In offspring embryos from WT line, PGCs were found in four cell clusters at 4-cell, Dome and 50\% epiboly stage (Fig. 2A, B and C). In offspring embryos from mutant line, PGCs were initially observed in four cell clusters at 4-cell stage (Fig. 2A), suggesting that the lineage specification of PGCs was not altered. Intriguingly, when
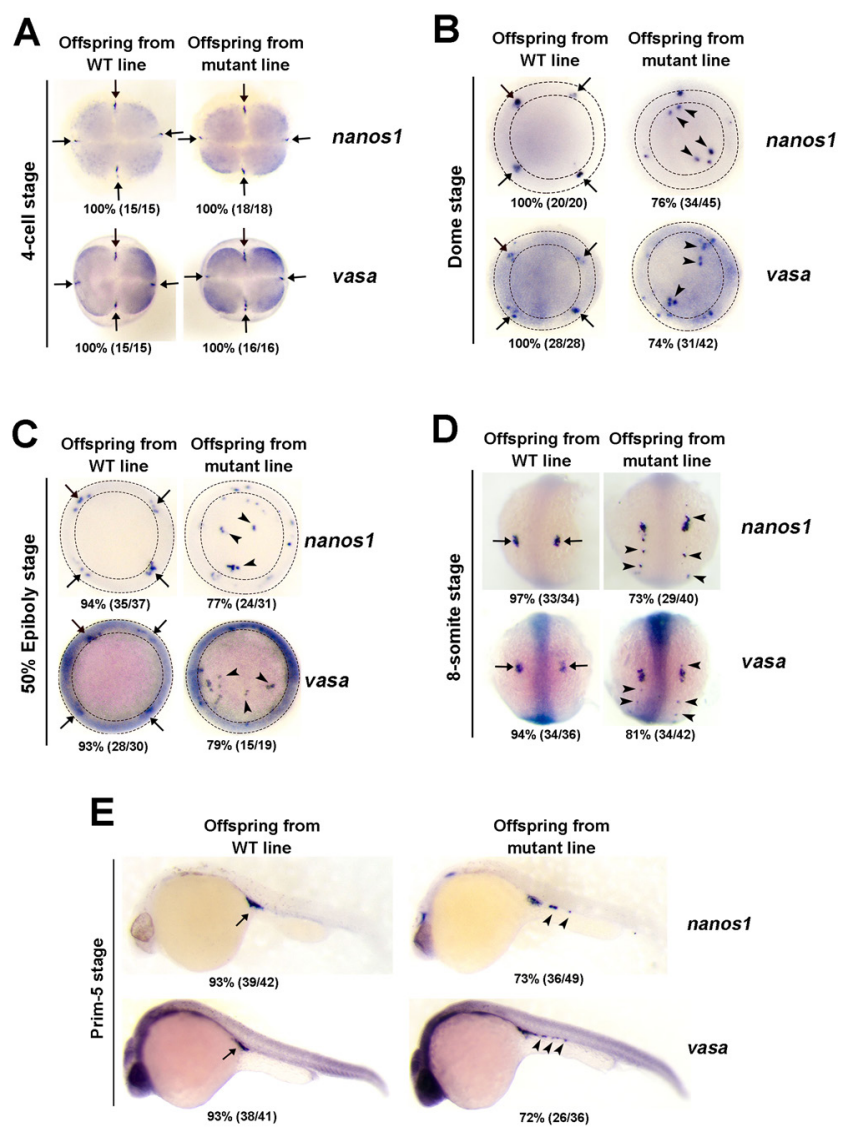

Figure $2 \mathrm{cftr}$ mutant induces nanos 1/vasa-marked PGCs disorder in early zebrafish embryo. Analysis of localization of nanos 1/vasa positive cells in offspring embryos from WT and mutant line by WISH at 4-cell stage (A), Dome stage (B), 50\% Epiboly stage (C), 8-somite stage (D) and Prim-5 stage (E). Embryo orientations: 4-cell, Dome stage and 50\% Epiboly stage, top view; 8-somite, dorsal view with anterior oriented at the top; Prim-5 stage, lateral views with anterior oriented toward the left. Arrows show the normal location of PGCs, arrowheads demonstrate the aberrant position of PGCs in cftr mutant. Region between two dotted circles on embryo shows normal location of PGCs. The numbers indicated in each picture are the number (left) of affected embryos with phenotype similar to what is shown in the picture and the total number (right) of observed embryos. The same number labeling was used thereafter.
PGCs enter the motile phase at Dome and 50\% epiboly stage, some PGCs were dispersed from the clusters, leading to ectopic localization of PGCs in $74-79 \%$ of offspring embryos from mutant line (Fig. $2 \mathrm{~B}$ and C). The ectopic localization of PGCs in $>72 \%$ of the offspring embryos from mutant line continued at 8-somite stage and Prim-5 stage, where some PGCs failed to migrate to the cell front and reach the genital ridges (Fig. 2D and $\mathrm{E}$ and Supplementary Table 1). These results suggested that Cftr is involved in regulating the migration of PGCs.

To verify the corresponding relation between PGCs phenotype and cftr-mutant genotype, we chose offspring embryos from mutant line with absent KV lumen at 8 -somite stage, and then detected vasa expression by $\mathrm{WISH}$, finally performed sequencing to investigate the genotype. Results indicated that embryos with absent KV lumen and disordered PGCs carried the $\mathrm{cttr}$ homozygous mutant (Supplementary Fig. 1). Interestingly, compared with WT fishes, more male adults were found in cftr homozygous mutant fishes even if breeding with low density (Supplementary Fig. 2), suggesting the depletion of PGCs in cftr homozygous mutant. These results were consistent with Tzung et al.'s discription (Tzung et al. 2015); they found that early depletion of PGCs or reduced PGCs number in zebrafish promotes testis formation and increases the percentage of male fishes.

\section{PGCs migration requires the ion channel function of CFTR}

Emerging evidence suggests CFTR can possess both ion channel function and non-ion channel function (Li \& Naren 2011). To validate the results from cftrmutant line and to examine the involvement of ion channel function of Cftr in PGCs migration, we treated the WT zebrafish embryos with CFTRinh-172, a potent and specific inhibitor of the CFTR channel that was identified by high-throughput screening (Ma et al. 2002, Taddei et al. 2004). CFTRinh-172 was added at $2.5 \mathrm{hpf}$ for $2 \mathrm{~h}$ and embryos were collected at 50\%-epiboly stage. PGCs were identified by WISH for nanos 1 and vasa as in previous experiment. The results showed that CFTRinh-172 dose dependently $(5-20 \mu \mathrm{M})$ distorted PGCs migration (Fig. 3). At $10 \mu \mathrm{M}$, a dose widely used to inhibit CFTR ion channel activity (Li et al. 2004), CFTRinh-172 distorted the migration of PGCs in 26-29\% of embryos (Fig. 3).

To further validate the involvement of $\mathrm{Cftr}$ ion channel function, we sought to restore the migration of PGCs by injecting WT or channel-defective ( $\triangle$ F508 and G551D) human CFTR mRNAs into the offspring embryos from mutant line. $\triangle \mathrm{F} 508$ mutation results in protein misfolding and retention in endoplasmic reticulum (ER) and negligible amount of the mutant CFTR reaching the plasma membrane, leading to impaired ion channel function (Amaral 2004). G551D is a well-known mutation causing gating defect in CFTR channel function 

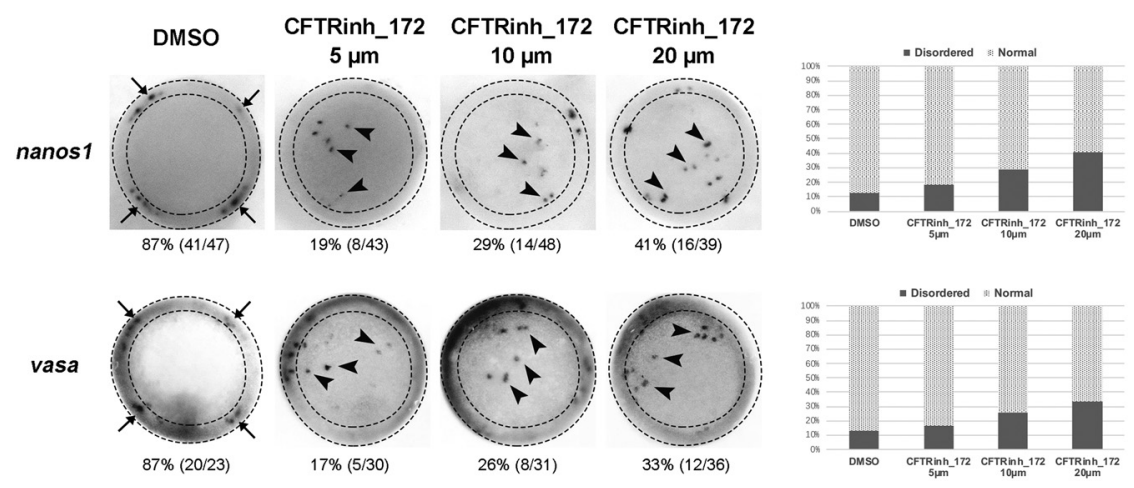

Figure 3 CFTR inhibitor CFTR_inh172 leads to PGCs disorder in early zebrafish embryo. Analysis of localization of nanos 1/vasa positive cells in embryos by WISH at $50 \%$ Epiboly stage with top view.
(Welsh \& Smith 1993, Hwang \& Kirk 2013). The results showed that WT CFTR mRNA markedly restored the migration of PGCs in offspring embryos from mutant line at $50 \%$ epiboly stage. Notably, both of the channeldefective mutants, $\Delta \mathrm{F} 508$ and G551D, failed to restore the localization of ectopically localized PGCs (Fig. 4 and Supplementary Table 2). Taken together, the results indicated that the involvement of Cftr in PGCs migration requires its ion channel activity.

\section{Elevation of cell motility factors in offspring embryos from mutant line}

To decipher the molecular mechanisms underlying the regulation of PGCs migration by $\mathrm{Cftr}$, we analyzed the expression of $c x c r 4 b, c x c / 12 a, \operatorname{rgs} 14 a$ and ca15b, key factors that account for the PGCs migration from dome stage to 8 -somite stage (Paksa \& Raz 2015), by RT-PCR (Fig. 5 and Supplementary Table 3). Cxcr4b is a chemokine receptor (Doitsidou et al. 2002) and distributed evenly around the cell perimeter along the PGCs migration route (Meyen et al. 2015), initiating an intracellular signaling cascade that biases the formation of protrusions for promoting PGCs migration. Chemokine Cxcl12a is a key attractant for mouse and zebrafish PGCs, which functions upon binding its receptor Cxcr4b in directing the cells to their target (Doitsidou et al. 2002, Molyneaux et al. 2003). The tight control over the spatiotemporal distribution of Cxcl12a in the embryo ensures an efficient PGC arrival at the target via a reproducible path while keeping the cells away from distant domains where the same chemokine is expressed (Mahabaleshwar et al. 2012). Rgs14a, the regulator of G-protein signaling 14a, is expressed in early PGCs germplasm (Hartwig et al. 2014), which participates in controlling the timing of PGCs migration initiation. Overexpressing Rgs14a in PGCs induces the formation of protrusions in all directions and the
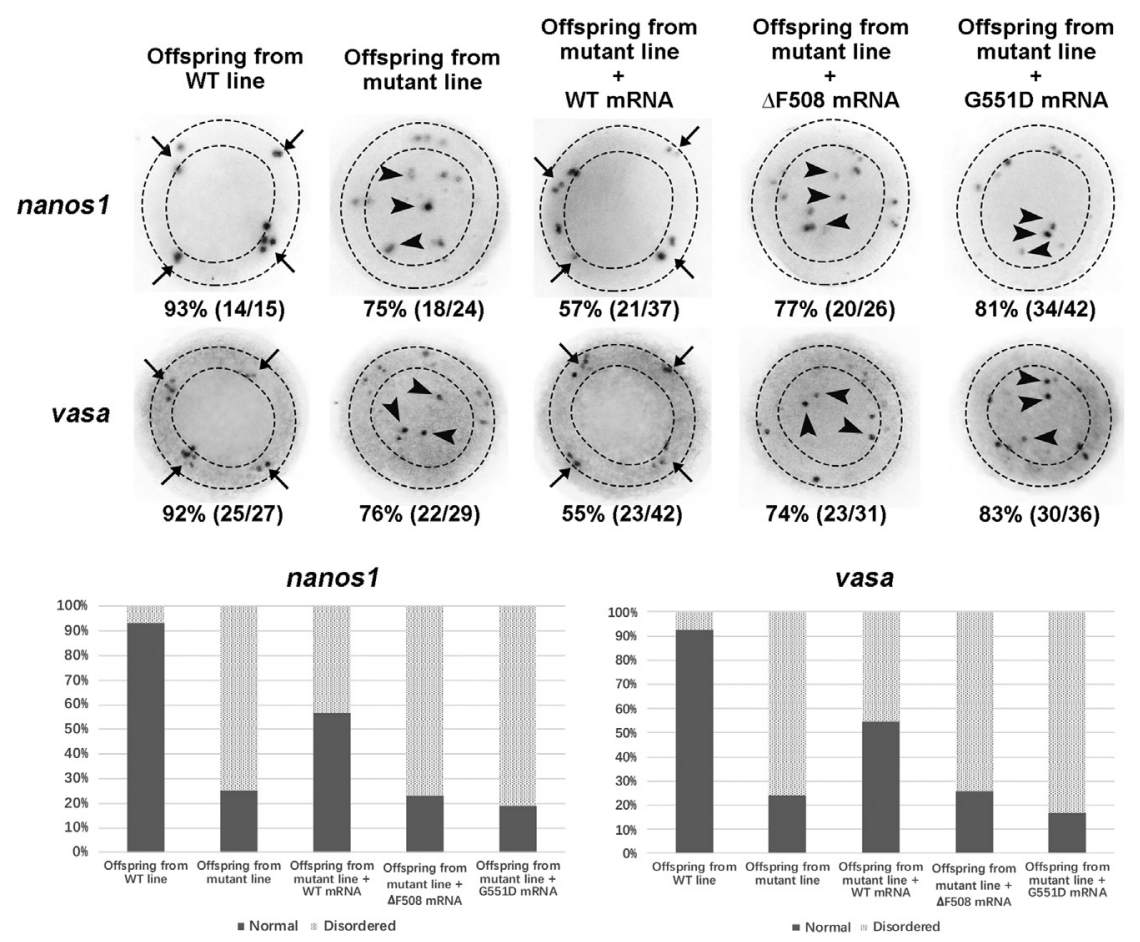

Figure 4 CFTR mutants with loss of ion channel function fail to recover PGCs development. Analysis of localization of nanos $1 /$ vasa positive cells in embryos by WISH at $50 \%$ Epiboly stage with top view. 

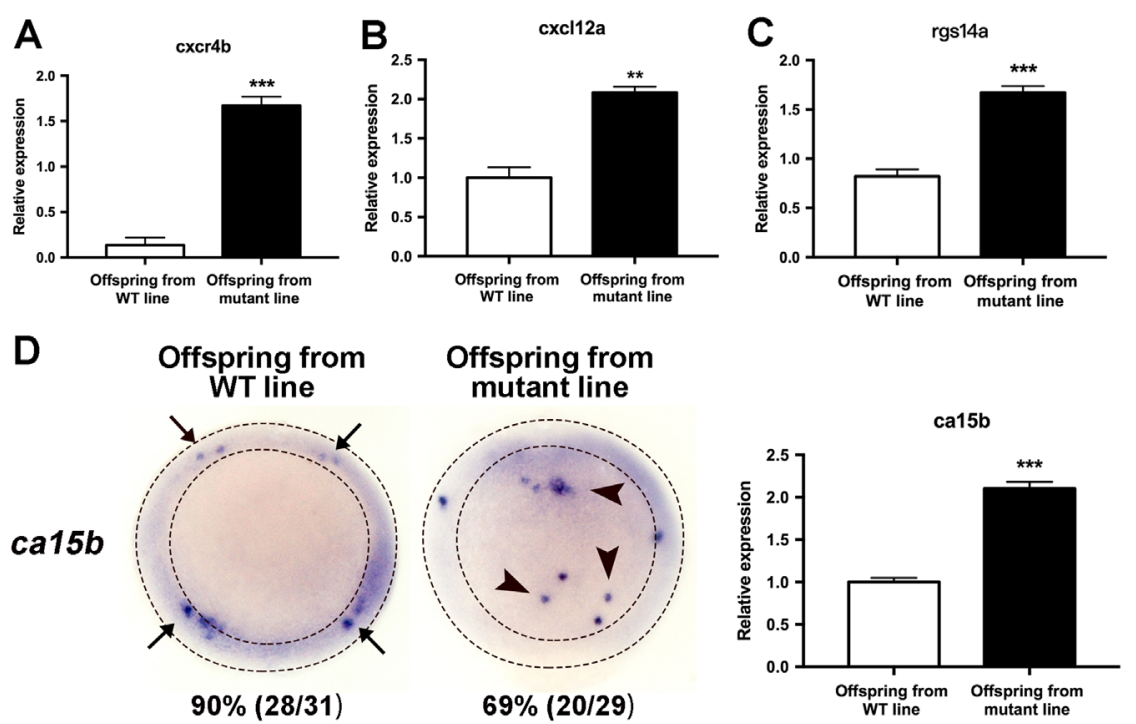

Figure 5 Aberrant expression of key factors (A: cxcr4b; B: cxcl12a; C: rgs14a; D: ca15b) is detected in offspring embryos from mutant line. 50\%-epiboly stage embryos were used in these assays. All of the factors were detected by qPCR; ca15b was also detected by WISH. reduction of motility relative to neighboring somatic cells (Hartwig et al. 2014). Ca15b (carbonic anhydrase $15 b)$, an enzyme that is expressed specifically in the PGCs, plays an important role in establishment of polar $\mathrm{pH}$ distribution for guided PGCs migration (Paksa \& Raz 2015, Tarbashevich et al. 2015). Our results showed that the expression of $c x c r 4 b, c x c / 12 a, \operatorname{rgs} 14 a$ and ca15b was significantly elevated in offspring embryos from mutant line (Fig. 5). Taken together, these results suggest that Cftr regulates PGCs migration through modulating the expression of factors involved in cell motility and response to chemotactic gradient in PGCs.

\section{Discussion}

CFTR is a well-known anion channel, and CFTR mutations are associated with CF and affect spermatogenesis in males (Xu et al. 2011). Besides the differentiated germ cells, the ion channel function of CFTR also plays an important role in embryo development (Lu et al. 2012, 2016). The demonstrated role of CFTR in primitive stem cells and germ cells prompted us to investigate the potential involvement of CFTR in regulating the development of the most primitive type of germ cells, the PGCs, in early embryogenesis.

Because of close relevance to mammals, zebrafish is a well-known model for uncovering molecular mechanism regulating PGCs development. To determine the role of cftr defects in PGCs development, Cas9/gRNA system was used to establish zebrafish cftr mutants. To minimize any off-target effects from the genome editing, mutant zebrafish lines were bred for three generations, and the F4 generation was used in this study. Nevertheless, a large percentage of homozygous cftr-mutant larvae was lost beginning around $10 \mathrm{dpf}$ (Navis \& Bagnat 2015); furthermore, fertility rate of adult homozygous cftr-/- fish was very low, which brought difficulty to experiment. Therefore, we used heterozygous cftr mutant line instead in this study, similar to our previous work (Sun et al. 2018).

Results showed that cftr mutants, both heterozygous $c f t r+/-$ and homozygous cftr-/-, led to ectopic locations of PGCs at different stages of zebrafish embryo development. Importantly, based on genotype result, all embryos carrying $\mathrm{cftr}+/$ - or $\mathrm{cttr}-/$ - mutant showed ectopic PGCs localization, suggesting the important and penetrant role of cftr in PGCs development. Since offspring embryos from mutant line with mixed genotypes have demonstrated significant change of PGCs and expression of key factors, which can reveal the key role of cftr in PGCs already, we did not persist in using homozygous $\mathrm{cftr}-\mathrm{l}-$ fishes.

Consistent with the previous report (Navis et al. 2013), our cftr homozygous mutant embryo also demonstrated the absence of KV lumen at 8-somite stage. In fact, except for $25 \%$ of offspring with absent KV, about $50 \%$ offspring embryos from mutant line showed reduced size of KV, which consistent with Roxo-Rosa et al.'s description (Roxo-Rosa et al. 2015), suggesting that these embryos are most likely the heterozygous cftr+/- mutants. RoxoRosa et al. found that injection of cftr AUG-morpholino to knock down Cftr expression severely impaired the lumen expansion of the KV, efficiently phenocopying cftr mutants. Furthermore, Navis et al. (Navis et al. 2013) also showed that their cftrpd1048 mutant, maybe a hypomorphic allele, demonstrated the severely reduced $\mathrm{KV}$ in size. Taken together, cftr plays an important role in controlling lumen expansion and function of $\mathrm{KV}$ in zebrafish.

Most interestingly, we found that male adult fishes were dominant in the cftr homozygous mutant fishes. This result is consistent with Tzung et al.'s report (Tzung et al. 2015), they find that the PGCs number is the key factor that control sex determination in zebrafish. Reduced PGCs will promote testis development and increase the proportion of male fishes in the offspring. 
In cftr mutant zebrafish, the number of PGCs that reached the genital ridges was reduced markedly, which resulted in increased male fishes in the cftr homozygous mutant offspring.

CFTR is a well-known anion channel, so we asked that whether PGCs migration was regulated by CFTR channel function. Firstly, we used CFTRinh-172, a CFTR channel gating inhibitor, to treat WT embryos, and found that CFTRinh-172 induced abnormal PGCs migration. Next, to get strong evidence of CFTR channel function on PGCs development, we injected mRNAs coding CFTR mutants with gating defect, including $\Delta \mathrm{F} 508$ and G551D, into offspring embryos from mutant line, respectively. Results showed that these two mutants, compared to WT mRNAs, failed to rescue the distorted migration of PGCs induced by cftr mutant. In brief, these results indicate the essential role of CFTR channel function in PGCs migration in early embryos.

Finally, we investigated the expression of key factors that control PGCs migration in offspring embryos from mutant line to understand the molecular mechanism of cftr mutant regulating PGCs migration (Paksa \& Raz 2015). These factors, including cxcr4b, cxcl12a, rgs $14 a$ and ca15b, control PGCs migration at 50\% epiboly stage. Results showed that the expression of all detected factors was increased significantly in cftr mutant embryos. These results indicate that CFTR defects have impact on the expression of chemokines.

According to Navis et al.'s description (Navis et al. 2013) and our previous study, CFTR is expressed ubiquitously during early embryogenesis (Sun et al. 2018), suggesting that CFTR may regulate PGCs migration by effect on both neighboring somatic cells and PGCs themselves. Our results show that genes both expressed specifically in PGCs, including rgs $14 a$ and ca15b, and somatic cells, including cxcr4b and cxc/12a, detected in this work are significantly elevated in offspring embryos from mutant line, suggesting that CFTR sustains PGCs migration through regulating key factors distributed at embryo widely. According to literature work, the precise molecular cascade linking $c x c r 4 b, c x c / 12 a, r g s 14 a$ and ca $15 b$ function is unknown so far (Tarbashevich et al. 2015), further investigation is needed in future work.

\section{Supplementary data}

This is linked to the online version of the paper at https://doi.org/10.1530/REP-17-0681.

\section{Declaration of interest}

The authors declare that there is no conflict of interest that could be perceived as prejudicing the impartiality of the research reported.

\section{Funding}

This research was supported by grants from National Natural Science Foundation of China (81200339), China Postdoctoral Science Foundation (20110491723), Young Teacher foundation of Sichuan University (2011SCU11040), National Basic Research Program of China (2012CB944903) and National 973 project (2013CB967404).

\section{Author contribution statement}

H S, X Z and H C C conceived and designed the experiments; H L, Y C, Z L, S X and M L performed the experiments; H S, X Z, Y L and H C C analyzed the data; H S, X Z and H C C wrote the paper.

\section{Acknowledgements}

The authors wish to thank Dr Kin Lam Fok (The Chinese University of Hong Kong) for helpful suggestions and careful modification.

\section{References}

Amaral MD 2004 CFTR and chaperones: processing and degradation. Journal of Molecular Neuroscience 23 41-48. (https://doi.org/10.1385/ JMN:23:1-2:041)

Chang N, Sun C, Gao L, Zhu D, Xu X, Zhu X, Xiong JW \& Xi JJ 2013 Genome editing with RNA-guided Cas9 nuclease in zebrafish embryos. Cell Research 23 465-472. (https://doi.org/10.1038/cr.2013.45)

Chen H, Guo JH, Lu YC, Ding GL, Yu MK, Tsang LL, Fok KL, Liu XM, Zhang XH, Chung YW et al. 2012 Impaired CFTR-dependent amplification of FSH-stimulated estrogen production in cystic fibrosis and PCOS. Journal of Clinical Endocrinology and Metabolism 97 923-932. (https://doi.org/10.1210/jc.2011-1363)

Doitsidou M, Reichman-Fried M, Stebler J, Koprunner M, Dorries J, Meyer D, Esguerra CV, Leung T \& Raz E 2002 Guidance of primordial germ cell migration by the chemokine SDF-1. Cell 111 647-659. (https:// doi.org/10.1016/S0092-8674(02)01135-2)

Gadsby DC, Vergani P \& Csanady L 2006 The ABC protein turned chloride channel whose failure causes cystic fibrosis. Nature 440 477-483. (https://doi.org/10.1038/nature04712)

Hartwig J, Tarbashevich K, Seggewiss J, Stehling M, Bandemer J, Grimaldi C, Paksa A, Gross-Thebing T, Meyen D \& Raz E 2014 Temporal control over the initiation of cell motility by a regulator of G-protein signaling. PNAS 111 11389-11394. (https://doi.org/10.1073/pnas.1400043111)

Hwang TC \& Kirk KL 2013 The CFTR ion channel: gating, regulation, and anion permeation. Cold Spring Harbor Perspectives in Medicine 3 a009498. (https://doi.org/10.1101/cshperspect.a009498)

Kimmel CB, Ballard WW, Kimmel SR, Ullmann B \& Schilling TF 1995 Stages of embryonic development of the zebrafish. Developmental Dynamics 203 253-310. (https://doi.org/10.1002/aja.1002030302)

Li C \& Naren AP 2011 Analysis of CFTR interactome in the macromolecular complexes. Methods in Molecular Biology 741 255-270. (https://doi. org/10.1007/978-1-61779-117-8_17)

Li H, Findlay IA \& Sheppard DN 2004 The relationship between cell proliferation, $\mathrm{Cl}$ - secretion, and renal cyst growth: a study using CFTR inhibitors. Kidney International 66 1926-1938. (https://doi.org/10.1111/ j.1523-1755.2004.00967.x)

Liu D, Wang Z, Xiao A, Zhang Y, Li W, Zu Y, Yao S, Lin S \& Zhang B 2014 Efficient gene targeting in zebrafish mediated by a zebrafish-codonoptimized cas9 and evaluation of off-targeting effect. Journal of Genetics and Genomics 41 43-46. (https://doi.org/10.1016/j.jgg.2013.11.004)

Liu Z, Guo J, Wang Y, Weng Z, Huang B, Yu MK, Zhang X, Yuan P, Zhao H, Chan WY et al. 2017 CFTR-beta-catenin interaction regulates mouse embryonic stem cell differentiation and embryonic development. 
Cell Death and Differentiation 24 98-110. (https://doi.org/10.1038/ cdd.2016.118)

Lu YC, Chen H, Fok KL, Tsang LL, Yu MK, Zhang XH, Chen J, Jiang X, Chung YW, Ma AC et al. 2012 CFTR mediates bicarbonate-dependent activation of miR-125b in preimplantation embryo development. Cell Research 22 1453-1466. (https://doi.org/10.1038/cr.2012.88)

Lu YC, Yang J, Fok KL, Ye YH, Jin L, Chen ZY, Zhang XM, Huang HF \& Chan HC 2016 Involvement of $\mathrm{Cl}(-) / \mathrm{HCO} 3(-)$ exchanger SLC26A3 and SLC26A6 in preimplantation embryo cleavage. Scientific Reports 6 28402. (https://doi.org/10.1038/srep28402)

Ma T, Thiagarajah JR, Yang H, Sonawane ND, Folli C, Galietta LJ \& Verkman AS 2002 Thiazolidinone CFTR inhibitor identified by highthroughput screening blocks cholera toxin-induced intestinal fluid secretion. Journal of Clinical Investigation 110 1651-1658. (https://doi. org/10.1172/JCI0216112)

Mahabaleshwar H, Tarbashevich K, Nowak M, Brand M \& Raz E 2012 beta-arrestin control of late endosomal sorting facilitates decoy receptor function and chemokine gradient formation. Development 139 2897-2902. (https://doi.org/10.1242/dev.080408)

Meyen D, Tarbashevich K, Banisch TU, Wittwer C, Reichman-Fried M, Maugis B, Grimaldi C, Messerschmidt EM \& Raz E 2015 Dynamic filopodia are required for chemokine-dependent intracellular polarization during guided cell migration in vivo. Elife $\mathbf{4}$ e05279. (https:// doi.org/10.7554/eLife.05279)

Molyneaux KA, Zinszner H, Kunwar PS, Schaible K, Stebler J, Sunshine MJ, O'Brien W, Raz E, Littman D, Wylie C et al. 2003 The chemokine SDF1/ CXCL12 and its receptor CXCR4 regulate mouse germ cell migration and survival. Development 130 4279-4286. (https://doi.org/10.1242/ dev.00640)

Navis A \& Bagnat M 2015 Loss of cftr function leads to pancreatic destruction in larval zebrafish. Developmental Biology 399 237-248. (https://doi.org/10.1016/j.ydbio.2014.12.034)

Navis A, Marjoram L \& Bagnat M 2013 Cftr controls lumen expansion and function of Kupffer's vesicle in zebrafish. Development 140 1703-1712. (https://doi.org/10.1242/dev.091819)

Nikolic A, Volarevic V, Armstrong L, Lako M \& Stojkovic M 2016 Primordial germ cells: current knowledge and perspectives. Stem Cells International 2016 1741072. (https://doi.org/10.1155/2016/1741072)

Paksa A \& Raz E 2015 Zebrafish germ cells: motility and guided migration. Current Opinion in Cell Biology 36 80-85. (https://doi.org/10.1016/j. ceb.2015.07.007)

Quinton PM 1999 Physiological basis of cystic fibrosis: a historical perspective. Physiological Reviews 79 S3-S22. (https://doi.org/10.1152/ physrev.1999.79.1.S3)

Raz E 2003 Primordial germ-cell development: the zebrafish perspective. Nature Reviews Genetics 4 690-700. (https://doi.org/10.1038/nrg1154)

Riordan JR 2008 CFTR function and prospects for therapy. Annual Review of Biochemistry 77 701-726. (https://doi.org/10.1146/annurev. biochem.75.103004.142532)

Roxo-Rosa M, Jacinto R, Sampaio P \& Lopes SS 2015 The zebrafish Kupffer's vesicle as a model system for the molecular mechanisms by which the lack of Polycystin-2 leads to stimulation of CFTR. Biology Open 4 1356-1366. (https://doi.org/10.1242/bio.014076)

Sang X, Curran MS \& Wood AW 2008 Paracrine insulin-like growth factor signaling influences primordial germ cell migration: in vivo evidence from the zebrafish model. Endocrinology 149 5035-5042. (https://doi. org/10.1210/en.2008-0534)
Snouwaert JN, Brigman KK, Latour AM, Malouf NN, Boucher RC, Smithies O \& Koller BH 1992 An animal model for cystic fibrosis made by gene targeting. Science 257 1083-1088. (https://doi.org/10.1126/ science.257.5073.1083)

Sun H, Li D, Chen S, Liu Y, Liao X, Deng W, Li N, Zeng M, Tao D \& Ma Y 2010 Zili inhibits transforming growth factor-beta signaling by interacting with Smad4. Journal of Biological Chemistry 285 4243-4250. (https://doi.org/10.1074/jbc.M109.079533)

Sun H, Wang Y, Zhang J, Chen Y, Liu Y, Lin Z, Liu M, Sheng K, Liao H, Tsang KS et al. 2018 CFTR mutation enhances Dishevelled degradation and results in impairment of Wnt-dependent hematopoiesis. Cell Death and Differentiation 9 275. (https://doi.org/10.1038/s41419-018-0311-9)

Taddei A, Folli C, Zegarra-Moran O, Fanen P, Verkman AS \& Galietta LJ 2004 Altered channel gating mechanism for CFTR inhibition by a highaffinity thiazolidinone blocker. FEBS Letters 558 52-56. (https://doi. org/10.1016/S0014-5793(04)00011-0)

Tarbashevich K, Reichman-Fried M, Grimaldi C \& Raz E 2015 Chemokinedependent $\mathrm{pH}$ elevation at the cell front sustains polarity in directionally migrating zebrafish germ cells. Current Biology 25 1096-1103. (https:// doi.org/10.1016/j.cub.2015.02.071)

Thisse C \& Thisse B 2008 High-resolution in situ hybridization to wholemount zebrafish embryos. Nature Protocols 3 59-69. (https://doi. org/10.1038/nprot.2007.514)

Tzung KW, Goto R, Saju JM, Sreenivasan R, Saito T, Arai K, Yamaha E, Hossain MS, Calvert ME \& Orban L 2015 Early depletion of primordial germ cells in zebrafish promotes testis formation. Stem Cell Reports $\mathbf{5}$ 156. (https://doi.org/10.1016/j.stemcr.2015.07.001)

Weidinger G, Wolke U, Koprunner M, Klinger M \& Raz E 1999 Identification of tissues and patterning events required for distinct steps in early migration of zebrafish primordial germ cells. Development $\mathbf{1 2 6}$ 5295-5307.

Weidinger G, Wolke U, Koprunner M, Thisse C, Thisse B \& Raz E 2002 Regulation of zebrafish primordial germ cell migration by attraction towards an intermediate target. Development 129 25-36.

Welsh MJ \& Smith AE 1993 Molecular mechanisms of CFTR chloride channel dysfunction in cystic fibrosis. Cell 73 1251-1254. (https://doi. org/10.1016/0092-8674(93)90353-R)

Westerfield M 1993 The Zebrafish Book. Oregon: University of Oregon Press.

Xu WM, Chen J, Chen H, Diao RY, Fok KL, Dong JD, Sun TT, Chen WY, Yu MK, Zhang XH et al. 2011 Defective CFTR-dependent CREB activation results in impaired spermatogenesis and azoospermia. PLoS ONE 6 e19120. (https://doi.org/10.1371/journal.pone.0019120)

Xu WM, Shi QX, Chen WY, Zhou CX, Ni Y, Rowlands DK, Yi Liu G, Zhu H, Ma ZG, Wang XF et al. 2007 Cystic fibrosis transmembrane conductance regulator is vital to sperm fertilizing capacity and male fertility. PNAS 104 9816-9821. (https://doi.org/10.1073/pnas.0609253104)

Received 6 November 2017

First decision 9 January 2018

Revised manuscript received 13 June 2018

Accepted 21 June 2018 